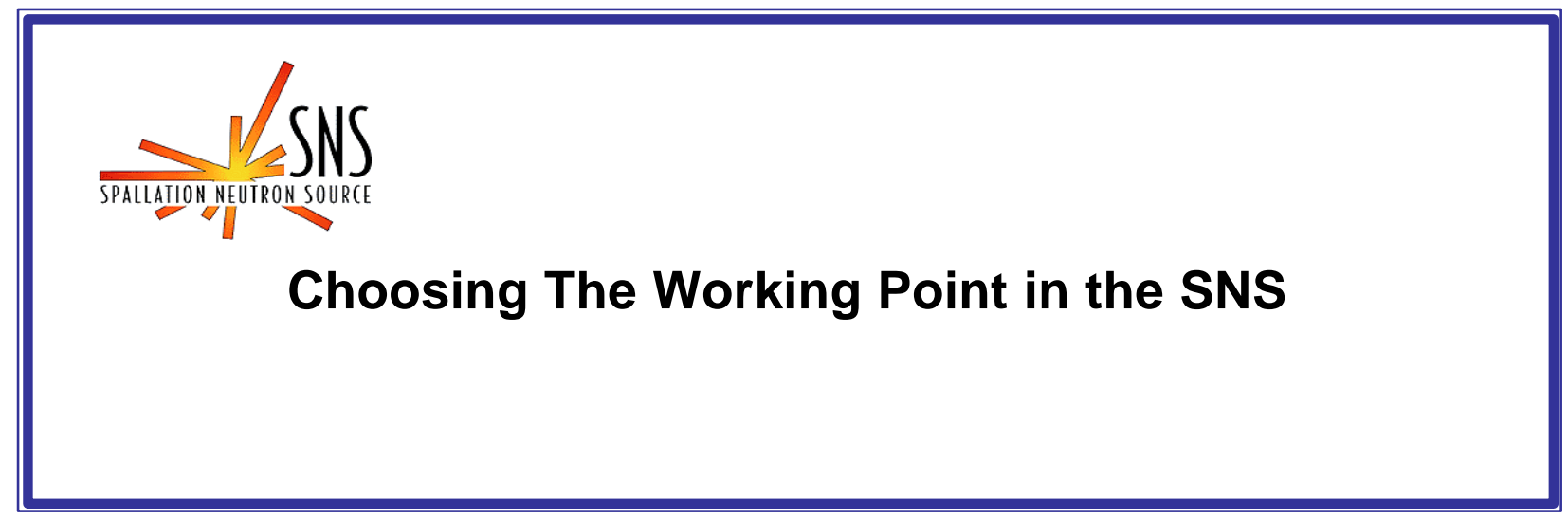

BNL/SNS TECHNICAL NOTE

NO. 090

G. Parzen

March 5, 2001

COLLIDER-ACCELERATOR DEPARTMENT

BROOKHAVEN NATIONAL LABORATORY

UPTON, NEW YORK 11973 


\title{
Choosing the Working point in the SNS
}

\author{
George Parzen
}

March 5, 2001

This note contains some suggestions for choosing the working point, $\nu_{x}, \nu_{y}$ for the SNS.

As a first step, one might make some assumptions about the resonances that one wants to avoid. Choosing the working point can be regarded as an iterative procedure, and the assumptions one makes in the first iteration can be corrected in the second iteration. Here, I assume that the resonances to be avoided include structure resonances up to and including the fourth order, and imperfection resonances up to and including the third order. I will also assume that only the sum resonances, and not the difference or coupling resonances, are to be avoided. There may be reasons why some coupling resonances should also be avoided, and this can be considered in the second iteration.

Having made these assumptions for the resonances to be avoided, one can now try to find the largest regions in $\nu_{x}, \nu_{y}$ which are free of these resonances. I will assume that these largest regions will be found along the $\nu_{x}=\nu_{y}$ coupling resonance. Much study of tune digrams showing all the resonance lines indicates that this is likely to be true. The possibility of splitting the tunes can be considered in the second iteration. Assuming that the largest resonance free regions will will be found along the $\nu_{x}=\nu_{y}$ coupling resonance, then these regions for the structure resonances are shown in Fig.1 for the SNS.

\section{Structure Resonances}

In Fig. 1, the tunes $\nu_{x}, \nu_{y}$ cover the range of 4 to 8 . The periodicity of the SNS is assumed to be 4 . The resonance lines that go through the points $\nu_{x}=\nu_{y}=4$ and $\nu_{x}=\nu_{y}=8$ include strong linear resonance which are 


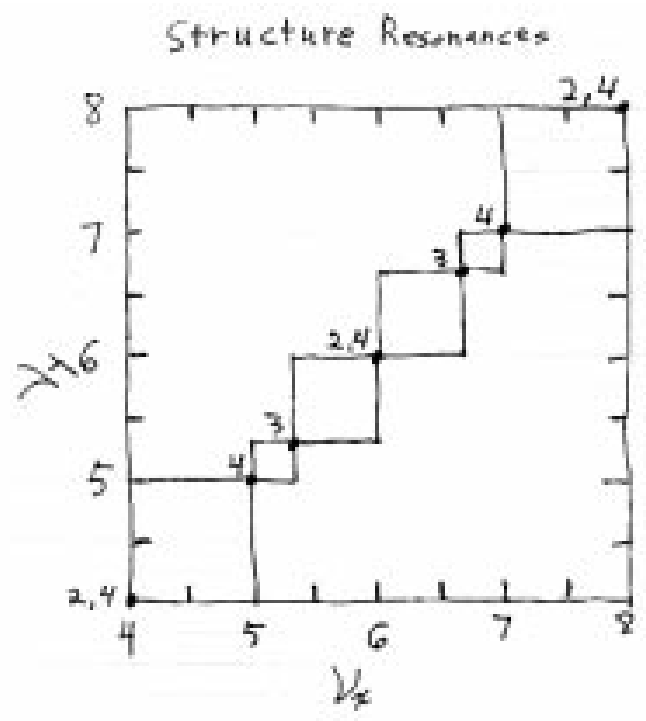

Figure 1: Structure resonances

usually avoided. The resonance free regions are shown by the squares along the $\nu_{x}=\nu_{y}$ line. The third order and fourth order structure resonances are indicated by dots on the $\nu_{x}=\nu_{y}$ line which are labeled 3 and 4 . The dot labeled 3 is the vertex of the third order sum resonances which can be considered to originate at this point and radiate outwards into the seond and fourth quadrants relative to this point. Similar remarks can be made regarding the dot labeled 4 which is the vertex for the fourth order sum resonance lines. The dot labeled 4 is also the vertex for the second order resonances. The largest resonance free squares are near the $\nu_{x}=\nu_{y}=4$ and $\nu_{x}=\nu_{y}=8$ points and are usually avoided because of their closeness to strong linear resonances. The largest remaining resonance free squares are the two found near the center of the figure. One square goes from $\nu_{x}=\nu_{y}=$ 5.3333 to $\nu_{x}=\nu_{y}=6$, and the second from $\nu_{x}=\nu_{y}=6$ to $\nu_{x}=\nu_{y}=6.6667$. These two squares have a width of $\Delta \nu=.6667$. This available resonance free tune width is considerably reduced by the imperfection resonances. 


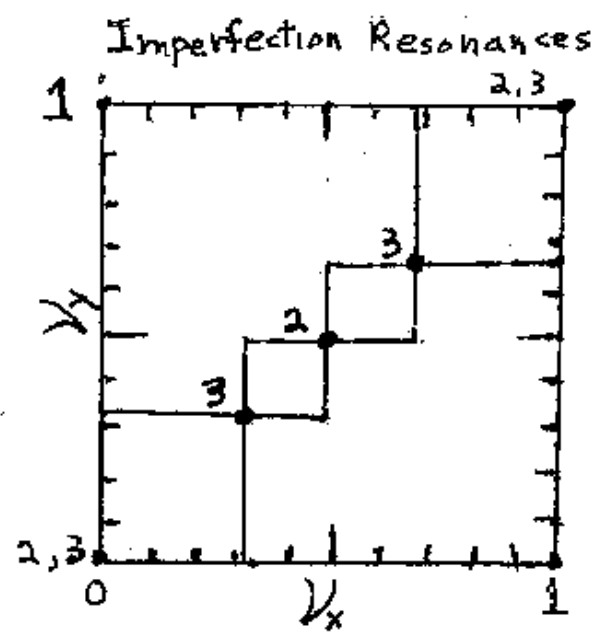

Figure 2: Imperfection resonances

\section{Imperfection Resonances}

Assuming that the largest resonance free regions will will be found along the $\nu_{x}=\nu_{y}$ coupling resonance, then these regions for the imperfection resonances are shown in Fig. 2 for the SNS. In Fig. 2, the tunes $\nu_{x}, \nu_{y}$ cover the range of 0 to 1 . The periodicity of the SNS for the imperfection resonances is 1 . The resonance free regions are shown by the squares along the $\nu_{x}=\nu_{y}$ line. The second order and third order imperfection resonances are indicated by dots on the $\nu_{x}=\nu_{y}$ line which are labeled 2 and 3. As for the structurs resonances, the dots may be considered as the vertices of the second order and third order imperfection resonances which radiate out from the vertices into the second and fourth quadrants relative to each vertex. In each unit square in $\nu_{x}, \nu_{y}$, the two largest resonance regions are found at the upper right and at the lower left of the unit square. Each of these squares has a width of $\Delta \nu=.3333$. Of the two squares, I prefer the upper right square, because in the SNS, space charge tends to move the tune downward, which is toward a third order imperfection resonance in the upper right square, and toward an imperfection integer resonance in the lower left square. 


\section{Combined Structure and Imperfection Reso- nances}

The two tune diagrams found above have now to be combined to show the resonance free regions when both structure resonances and imperfection resonances are present. This is shown in Fig. 3. In Fig. 3, the $\nu_{x}, \nu_{y}$ space is shown from $\nu_{x}=\nu_{y}=5$ to $\nu_{x}=\nu_{y}=7$. The vertex of each set of resonances is shown as a dot on the nux=nuy line. The numbers 3 and 4 indicate the third and fourth order structure resonances, while $\hat{2}$, and $\hat{3}$ indicate second and third order imperfection resonances. The two largest and preferred regions are the square that goes from $\nu_{x}=\nu_{y}=5.6667$ to $\nu_{x}=\nu_{y}=6.0000$ and the square that goes from $\nu_{x}=\nu_{y}=6.6667$ to $\nu_{x}=\nu_{y}=7.0000$. These squares have a width of $\Delta \nu=.3333$ which is to be compared with the roughly $\Delta \nu=.2$ spread expected from space charge. Without doing any simulation studies, one can hazard a guess for the possible linear operating tune as either $\nu_{x}=5.900, \nu_{y}=5.895$ or $\nu_{x}=6.900, \nu_{y}=6.895$. The $\nu_{y}$ has been lowered by .005 to move off the $\nu_{x}=\nu_{y}$ coupling resonance. A more accurate location of the linear operating tune could be found by doing a simulation study, using the above tune guess, to find the actual spread in the tune. My so far preferred working point is indicated in Fig. 3 by a cross.

\section{Further Iterations}

The first iteration found a largest resonance free region whose width was $\Delta \nu=.3333$ compared to the roughly $\Delta \nu=.2$ spread due to space charge. This indicates that there is not much leeway for further restrictions on the choice of the working point.

Moving off the $\nu_{x}=\nu_{y}$ line, splitting the tunes, will probably reduce the size of the available resonance free regions, and this has to be balanced against whatever one thinks one gains by splitting the tunes.

Trying to avoid even higher order resonances than fourth order structure resonance and the third order imperfection resonance will probably reduce the size of the available resonance free regions, and there is not much room for this reduction.

A possible error in the considerations and results of the first itereation is the assumption that the lower order resonances, structure resonances up to and including the fourth order and imperfection resonances up to and in- 
cluding the third order, need to be avoided. Simulation studies may indicate that some of these lower order resonances are not dangerous. However, one may still want to avoid these lower order resonances if it is possible to avoid them. 
Structure and Imperfection Resonances

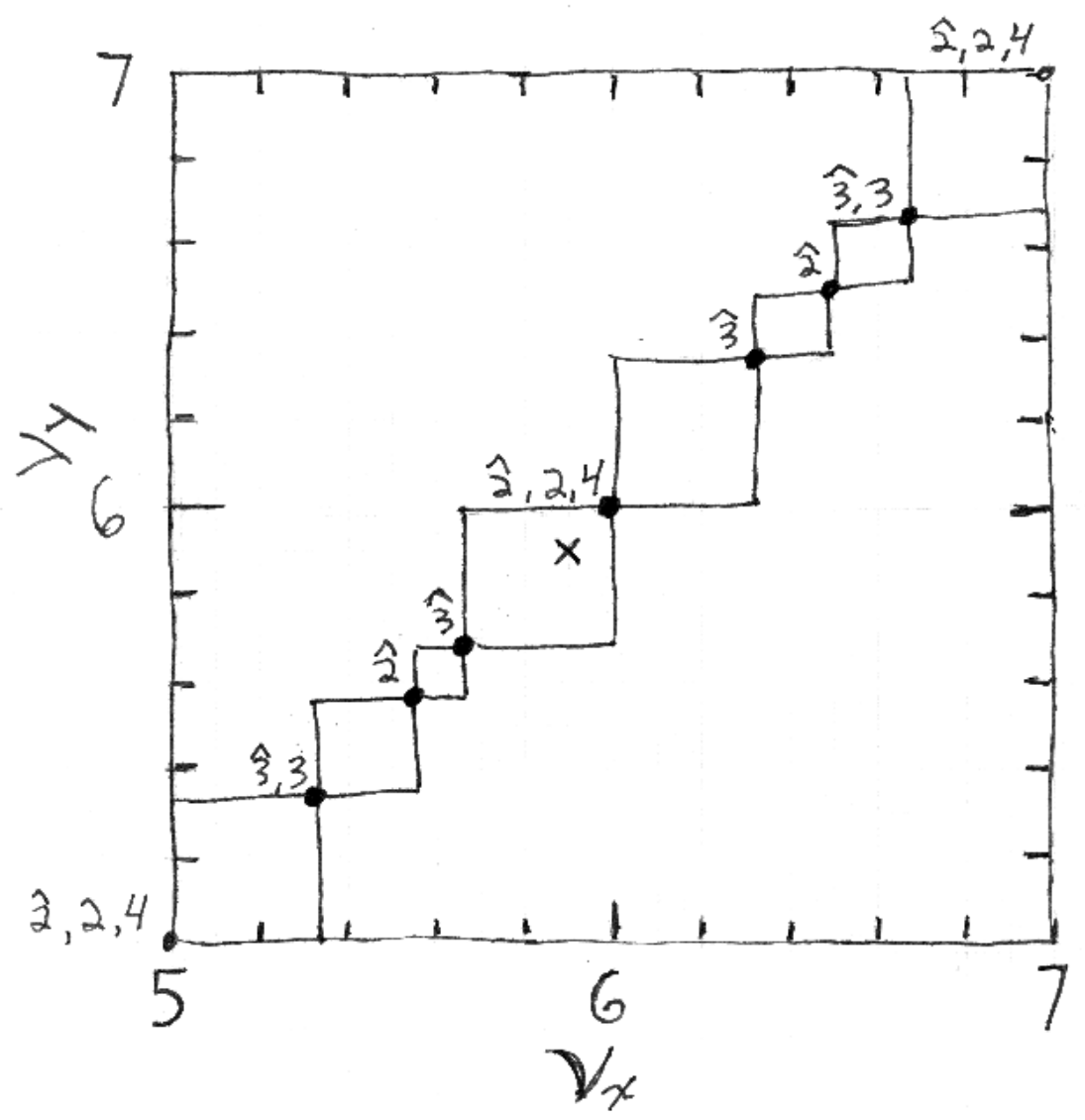

Figure 3: Combined structure and imperfection resonances. The numbers $2,3,4$ indicate structure resonaces while $\hat{2}, \hat{3}$ indicate imperfection resonances. A possible working point is indicated by the cross. 